WORK IN PROGRESS

\title{
Smart pens to assist fibre optic sensors research: Evaluating OCR tools
}

\author{
Anne Dozias, Cristian Camilo Otalora-Leguizamón, \\ Marco Bianchetti, Maria Susana Avila-Garcia
}

Published: 31 October 2018

\begin{abstract}
Reproducibility is one of the big challenges in research. Lab notebooks have been used to record data, observations and relevant remarks of the research processes. Smart pens are devices that record audio, handwriting notes thanks to micro patterned paper, and generate pdf files and audio enriched notes (pencasts). The handwriting notes can then be processed using optical character recognition (OCR) software to generate digital documents allowing the user to archive and access these notes in an easier way. However, OCR for handwriting is still a challenge in the computer vision research area. In this paper, we report the evaluation results of different OCR tools when processing handwriting notes written by 7 participants focusing on the main elements and technical vocabulary identified in fibre optic sensors research.
\end{abstract}

Keywords: Electronic Laboratory Notebook; Reproducibility; Smart Pen; Experimental Research; Evaluation.

\section{Introduction}

Lab notebooks are used to register experimental design data, observations, results, etc. Recording scientific notes is a very important activity in research environments so that reproducibility can be achieved, and an organised access to such notes is quite important. Electronic Laboratory Notebooks have been proposed in various areas of research such as chemistry [11] and neuroscience [12].

In this paper we focus on Fibre Optic Sensors research, specifically on how scientific notes are registered and in which way this contributes to reproducibility. Smart pens are devices that offer the functionality of registering handwriting notes in special micro patterned notepads and generating digital documents that can be archived. Although these documents preserve as an image the handwriting notes, there are optical

\section{Dozias, A.}

ISEN Brest

20 Rue Cuirassé Bretagne, 29200 Brest, France

Email: anne.dozias@hotmail.fr

Otalora-Leguizamon, CC., Bianchetti, M., Avila-Garcia, MS.

Universidad de Guanajuato

Yurira, Guanajuato.

Email: cc.otaloraleguizamon@ugto.mx., mb@ugto.mx,

susana.avila@ugto.mx. character recognition (OCR) software applications that allow converting those images into text, facilitating the access to them by applying data mining techniques, extracting data that can help to classify the information contained.

OCR is used for data mining in different areas of research, for instance for studying historical texts in medicine, where the challenges include the poor quality of the images generated [9]. Furthermore, there are proposals for classifying OCR errors, such as the work reported in [10] that reported a variation of a dynamic programming algorithm applied to characters in three different fonts. Image to text transcription is a challenge in computer vision given that scanned files introduce errors that may be hard to solve, therefore, researchers are proposing different approaches using statistical and mathematical methods. For instance, in [8] authors propose a method based on statistical learning using historical biology data, as a solution to specific error patterns of OCR solutions. This work is focused on evaluating existing OCR solutions in fibre optic sensors scientific research notes.

\section{Use of logbooks in fibre optics sensor research}

We interviewed six researchers working in fibre optics research. We asked for examples of the lab notes they take to identify the information registered. Three main elements were identified in the lab notes:

- Text notes, including data elements such as optic fibre type, sensor structure type, descriptions, comments, dates, time, common measurements, used equipment, sensor assay name

- Diagrams, sketches, graphics, and

- Tables with the interferometers manufactured, their characteristics and parameters.

Considering these information elements, an experiment described in the next section was carried out.

\section{Experimental design}

\subsection{Tools}

The following tools were used in this experiment:

- A livescribe notebook,

- Three echo smartpen

- A computer, Windows 8 Operating System

- Echo Desktop software

OCR software (using the free trial period): PaperPort, ABBYY, Adobe Acrobat DC, Readiris 17 and MyScript. 

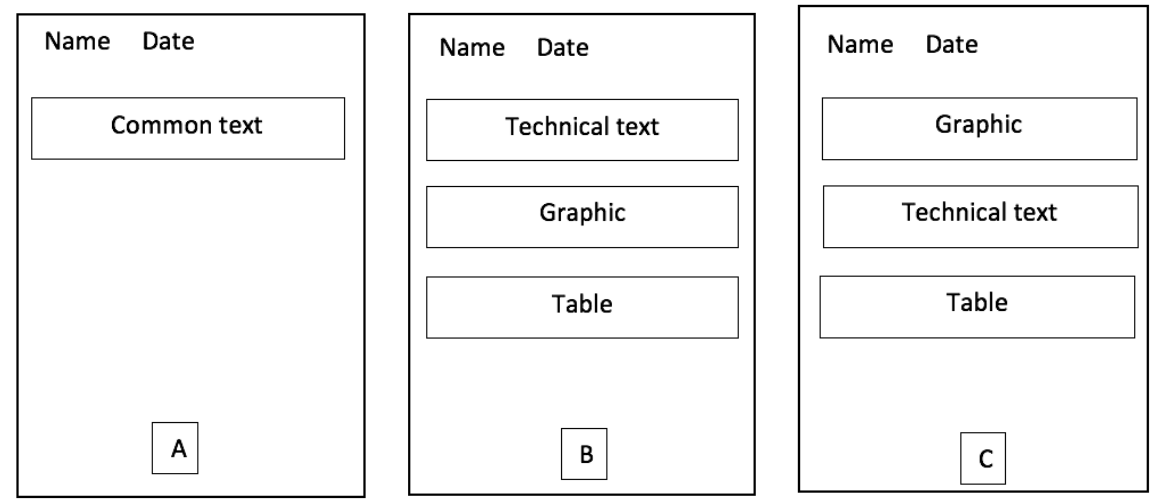

Figure 1. Illustration of the page format configurations reproduced by participants

Table 1. Results obtained in average for all participants

\begin{tabular}{|c|c|c|c|c|c|c|}
\hline & $\begin{array}{l}\text { Quality of } \\
\text { writing based } \\
\text { on the ease of } \\
\text { reading, the } \\
\text { crossing-out }\end{array}$ & $\begin{array}{c}\text { Word } \\
\text { accuracy } \\
\text { technical } \\
\text { text (51 } \\
\text { words) } \\
\text { Mean value }\end{array}$ & $\begin{array}{c}\text { Word } \\
\text { accuracy } \\
\text { common } \\
\text { text }(27 \\
\text { words) } \\
\text { Mean } \\
\text { value }\end{array}$ & $\begin{array}{c}\text { Date } \\
(09 / 07 / 2018)\end{array}$ & $\begin{array}{c}\text { Quality of } \\
\text { graphic and } \\
\text { table based on } \\
\text { the } \\
\text { resemblance } \\
\text { with the } \\
\text { original }\end{array}$ & $\begin{array}{l}\text { Accuracy of the conversion for the } \\
\text { table and graphic }\end{array}$ \\
\hline MyScript & Good & $49 / 51$ & $27 / 27$ & Transcribed & Good & $\begin{array}{l}\text { Only some words are accurate, not } \\
\text { all of them. The lines and arrows are } \\
\text { not transcribed. }\end{array}$ \\
\hline PaperPort & Good & $0 / 51$ & $0 / 27$ & Not transcribed & Good & Not transcribed \\
\hline Readiris & Good & $0 / 51$ & $0 / 27$ & Not transcribed & Good & Not transcribed \\
\hline ABBYY & Good & $0 / 51$ & $0 / 27$ & Not transcribed & Good & Not transcribed \\
\hline Adobe Acrobat DC & Good & $0 / 51$ & $0 / 27$ & Not transcribed & Good & Not transcribed \\
\hline
\end{tabular}

\subsection{Participants}

Seven university students were recruited as participants to carry out the activities.

\subsection{Activities}

Following the information obtained from the researchers, three activities were designed for each participant. All the three activities were performed with a Livescribe Echo SmartPen on a special micro patterned paper notebook on three different configurations as shown in Figure 1. In the first activity, participants wrote a common text. In the second one, participants wrote a technical text, draw a graph, and designed a table. In the third activity, the content of the text, graph, and table were unchanged with respect to those in the second activity; however, the order of the graph and of the technical text were inverted while the table remained in third position. The change in order was defined to explore how figures and tables are processed by the OCR software applications and whether order makes a difference

\subsection{Content to reproduce}

The first text, called Common Text is a simple one with common vocabulary with a proper noun (Guanajuato and Mexican), numbers and punctuation (point and comma). It is 27 words long (punctuation included). The text was extracted from [1].
"The university is based in the Mexican state of Guanajuato, made up of about 33,828 students in programs ranging from high school level to doctorate level."

The second text, referred as Technical Text, contains technical vocabulary, numbers, punctuation (point, hyphen and parentheses). It is 51 words long (punctuation included). The text was extracted from [2].

"The simplest type of optical fiber is called single-mode. It has a very thin core about 5-10 microns (millionths of a meter) in diameter. In a single-mode fiber, all signals travel straight down the middle without bouncing off the edges"

The table to reproduce was extracted from [3] and the graphic from [4].

\section{OCR Software evaluation}

Figure 2 shows a sample of the handwritten notes of one participant produced following format B of the second activity (see Figure 1). The handwriting notes produced were downloaded from the pens using the Echo Desktop Application. Images for the notes were produced to be used in the OCR software evaluation, except for MyScript which is already linked to Echo Desktop application [5], so it was not necessary to convert the data to pdf. 


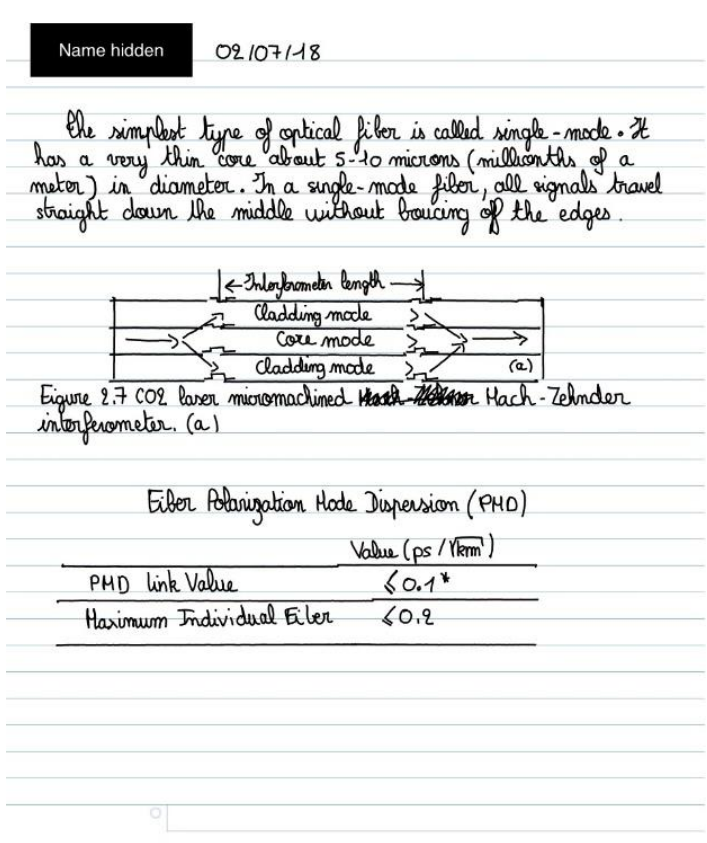

Figure 2. Notes sample showing the handwriting notes of the technical text of the second activity as illustrated in Figure 1.

\section{Results}

The results of the evaluation were transcribed in a table that takes into an account the following elements: the words accuracy, the quality of the graphic and table produced, the quality of the handwriting, the speed of the transcription.

For PaperPort, Adobe Acrobat DC, Readiris and ABBYY the table shows that no word has been rightly transcribed, and neither were the graphic and table. For MyScript, the transcription of words was way more efficient with almost every word translated accurately but graphics and table were not reproduced correctly as it can be seen in Table 1 .

\section{Conclusions and future work}

Results show that just one of the OCR software was providing good results, but only when transcribing words in sentences. OCR software applications were not able to identify graphics and tables. In this experiment, MyScript stands out because it was not necessary to convert the data to a pdf format for transcription obtained from the other OCR software applications.

It is important to note that in this experiment the conversion of the notes to pdf decreased the quality of the file which may have be an important factor that influenced the results. Further tests need to be carried out to test the OCR software applications again with better quality generated pdf files for transcription. Recently, we carried out a pdf conversion using a Mac Air laptop and the conversion results were better in quality.

The results obtained from myScript show that it may not be possible to recognise graphics and table content and generate the associated metadata. Reading the content of the notes and its associated elements is important to be able to organise scientific notes in an information system automatically. We will look for other alternatives to label the elements to automatically describe them.

\section{Acknowledgments}

We thank all the participants for their time. Anne Dozias thanks to ISEN Brest and JALI for the grant provided to carry out her research internship at the University of Guanajuato. Camilo Otalora is supported by the CONACYT grant $n^{\circ} 828846$. This work was supported by the SEP-PRODEP grants UGTO-PTC-510 y UGTO-PTC-512.

\section{References}

[1] Bird, C. L., Willoughby, C., \& Frey, J. G. (2013). Laboratory notebooks in the digital era: the role of ELNs in record keeping for chemistry and other sciences. Chem. Soc. Rev, 42, 8157. https://doi.org/10.1039/c3cs60122f

[2] Chris Woodford. June 14, 2018. Fiber Optics from https://www.explainthatstuff.com/fiberoptics.html

[3] Corning SMF-28 Optical Fiber Product Information Issue of August 2002.

[4] Echo Desktop https://www.livescribe.com/en/support/echo/setup: Echo Desktop. Inc.2007

[5] J. Esakov, D. P. Lopresti, and J. S. Sandberg, "Classification and distribution of optical character recognition errors," Proc. SPIE --- Int. Soc. Opt. Eng., vol. 2181, no. March 1994, pp. 204-216, 1994.

[6] J. Mei, A. Islam, A. Moh'd, Y. Wu, and E. Milios, "Statistical learning for OCR error correction," Inf. Process. Manag., vol. 54, no. 6, pp. 874-887, 2018.

[7] P. Thompson et al., "Text mining the history of medicine," PLoS One, vol. 11, no. 1, pp. 1-33, 2016.

[8] Schwartz, M. Guidelines for Bias-Free Writing.

[9] Indiana University Press, Bloomington, IN, USA, 1995

[10] Trefethen, A., Roure, D. De, Newman, D., Wallom, D., Saddy, D., Smith, G., ... Connor, V. O. (n.d.). NeuroHub : A Research Information Environment for Neuroscientists, 3-5.

[11] Wikipedia Page of the University of Guanajuato https://en.wikipedia.org/wiki/Universidad_de_Guanajuato

[12] Yaping-Xu. 2013. Multi-parameter sensing based on in-line Mach-Zehner interferometer. Faculty of Graduate and Postdoctoral Studies in partial fulfilment of the requirement for the M.Sc. degree in Physics.

[13] Zellweger, P.T., Bouvin, N.O., Jehøj, H., and Mackinlay, J.D. Fluid Annotations in an Open World. Proc. Hypertext 2001, ACM Press (2001), 9-18. 